\title{
Cine CT for Attenuation Correction in Cardiac $\mathrm{PET} / \mathrm{CT}$
}

\author{
Adam M. Alessio ${ }^{1}$, Steve Kohlmyer ${ }^{2}$, Kelley Branch ${ }^{3}$, Grace Chen $^{3}$, James Caldwell ${ }^{1,3}$, and Paul Kinahan ${ }^{1}$ \\ ${ }^{1}$ Department of Radiology, University of Washington, Seattle, Washington; ${ }^{2}$ GE Healthcare, Waukesha, Wisconsin; and ${ }^{3}$ Division of \\ Cardiology, University of Washington, Seattle, Washington
}

In dual-modality PET/CT systems, the CT scan provides the attenuation map for PET attenuation correction. The current clinical practice of obtaining a single helical CT scan provides only a snapshot of the respiratory cycle, whereas PET occurs over multiple respiratory cycles. Misalignment of the attenuation map and emission image because of respiratory motion causes errors in the attenuation correction factors and artifacts in the attenuationcorrected PET image. To rectify this problem, we evaluated the use of cine CT, which acquires multiple low-dose CT images during a respiratory cycle. We evaluated the average and the intensity-maximum image of cine CT for cardiac PET attenuation correction. Methods: Cine CT data and cardiac PET data were acquired from a cardiac phantom and from multiple patient studies. The conventional helical CT, cine CT, and PET data of an axially translating phantom were evaluated with and without respiratory motion. For the patient studies, we acquired 2 cine CT studies for each PET acquisition in a rest-stress ${ }^{13} \mathrm{~N}$-ammonia protocol. Three readers visually evaluated the alignment of 74 attenuation image sets versus the corresponding emission image and determined whether the alignment provided acceptable or unacceptable attenuation-corrected PET images. Results: In the phantom study, the attenuation correction from helical CT caused a major artifactual defect in the lateral wall on the PET image. The attenuation correction from the average and from the intensity-maximum cine CT images reduced the defect by $20 \%$ and $60 \%$, respectively. In the patient studies, $77 \%$ of the cases using the average of the cine CT images had acceptable alignment and $88 \%$ of the cases using the intensity maximum of the cine CT images had acceptable alignment. Conclusion: Cine CT offers an alternative to helical CT for compensating for respiratory motion in the attenuation correction of cardiac PET studies. Phantom studies suggest that the average and the intensity maximum of the cine CT images can reduce potential respirationinduced misalignment errors in attenuation correction. Patient studies reveal that cine CT provides acceptable alignment in most cases and suggest that the intensity-maximum cine image offers a more robust alternative to the average cine image.

Key Words: PET/CT; 4DCT; attenuation correction; cardiac imaging; respiratory motion

J Nucl Med 2007; 48:794-801

DOI: 10.2967/jnumed.106.035717

Received Aug. 18, 2006; revision accepted Dec. 3, 2006.

For correspondence or reprints contact: Adam M. Alessio, PhD, Department of Radiology University of Washington, Box 357987, Old Fisheries Center,

Room 200, 4000 15th Ave. NE, Seattle, WA 98195-7987.

E-mail: aalessio@u.washington.edu

COPYRIGHT @ 2007 by the Society of Nuclear Medicine, Inc.
$\mathbf{P}$ ET combined with $\mathrm{CT}$ in an integrated PET/CT scanner offers a single-study, noninvasive technique for the diagnosis of coronary artery disease. The integrated modalities provide distinct and complementary information: PET offers functional measurement of myocardial perfusion and metabolism, whereas contrast-enhanced CT angiography offers structural assessment of coronary anatomy and atherosclerotic burden $(1,2)$.

A limitation with cardiac PET on a PET/CT system is that the PET attenuation map formed from a helical CT acquisition represents a snapshot of the respiratory cycle, whereas the PET image is acquired over multiple respirations. The potential attenuation and emission misalignment dramatically reduces the accuracy of attenuation correction, leading to serious artifacts in the PET image in a large fraction of studies.

The effects of respiratory motion on attenuation-emission scan mismatch with PET/CT scanners has received growing interest in the past few years and has been explored for both oncologic and cardiac applications. Simulation studies of dynamic phantoms have shown errors of as much as $\pm 25 \%$ on attenuation-corrected PET images from respiratory motion-mismatched CT acquisitions (3). Chin et al. showed in a canine study that CT performed at end-exhalation or end-inhalation results in PET myocardial regional errors on the order of $\pm 6 \%$ (4). They also showed that misregistration along the diaphragm causes global quantitation errors on the PET image and erroneous heterogeneity in the myocardium. Goerres et al. demonstrated that mismatches can cause mean errors of up to $\pm 35 \%$ in clinical human studies comparing CT attenuation correction to conventional PET transmission scans with germanium sources (5). Several groups have explored the option of performing the CT scan at an optimal time during respiration, such as at mid expiration, to minimize potential mismatches (6-8). Our clinical experience has found that asking patients to hold their breath at a certain point in their respiratory cycle causes highly variable and often undesirable results. Even with perfect breath coaching, it is difficult to state the optimal breath protocol applicable for all patients (mid expiration vs. full expiration vs. shallow expiration vs. constant shallow breathing). And finally, even if the CT 
scan is obtained at the optimal location, it will not perfectly match the PET scan acquired over many full respiratory cycles.

Figure 1 presents an axial and coronal slice from a ${ }^{13} \mathrm{~N}$ ammonia $\left({ }^{13} \mathrm{NH}_{3}\right)$ stress study performed on a GE Discovery STE PET/CT scanner. The left column shows images from a helical CT scan used for attenuation correction of the PET images in the right column. The same line in 3-dimensional space is drawn from the edge of the cardiac lateral wall on the $\mathrm{CT}$ scan to the edge of the wall on the PET scan, marking the almost $2.5-\mathrm{cm}$ mismatch between the attenuation and emission images. This mismatch leads to the artifactual defect highlighted on the same PET slices.

We proposed using cine CT acquisition, and derived images, to reduce attenuation correction errors from respiration and cardiac motion. Cine $\mathrm{CT}$ essentially acquires multiple low-dose CT scans over time at each slice in the patient. Pan et al. have demonstrated the efficacy of averaged cine CT images for oncologic imaging (9). Cook et al. have presented initial results on the benefits of averaged cine CT images for cardiac imaging in a canine study (10). We demonstrated through phantom studies and our initial clinical patient studies the efficacy of attenuation correction with 2 combinations of cine CT images: average images and intensity-maximum images.

Cardiac PET with the radiotracer ${ }^{18} \mathrm{~F}-\mathrm{FDG}$ is considered the gold-standard indicator of myocardial viability (11-13). PET studies with either ${ }^{82} \mathrm{Rb}$ or ${ }^{13} \mathrm{NH}_{3}$ offer the potential for quantitative myocardial perfusion assessment (14-17). Moreover, gated cardiac PET with these tracers has been shown to assess left ventricular function well (18). These cardiac PET techniques provide valuable information regarding the diagnosis of ischemia or infarction and offer prognostic information to guide patient treatment.

High-energy-photon attenuation correction factors for PET are derived from a transmission scan. In conventional stand-alone PET systems, this transmission scan is acquired over a period of about 3-20 min with a rotating external rod source (germanium or cesium), providing a noisy attenua- tion map of the patient. In modern, integrated PET/CT systems, the CT scan is transformed to provide a fast, relatively noise-free attenuation map (19). If the attenuation image is misaligned with the emission image, the attenuation correction factors are incorrect and cause artifacts in the attenuation-corrected PET image. With cardiac imaging, there is a strong attenuation gradient along the myocardial free wall, with muscle next to the air of the lung space. Another strong attenuation gradient occurs along the top of the right diaphragm, which, for many patients, enters and leaves the same axial slice as does the left ventricle. If misalignments occur along these boundaries, the attenuation correction factors are potentially highly inaccurate, causing as much as a 60\% error in the PET tracer emission image in the critical regions of diagnostic interest (20). Artifacts caused by misalignment are particularly disconcerting in cardiac imaging because they can present themselves as perfusion abnormalities or erroneous information on myocardial viability (21).

The American Society of Nuclear Cardiology warns against these potential attenuation correction-induced artifacts in stand-alone PET and PET/CT systems (22). McCord et al. have shown that even a small, 2-cm, shift between transmission and emission images can cause up to a $30 \%$ myocardial regional increase or decrease on the PET image, especially in the free wall (20), leading to qualitative and quantitative misinterpretations. These emissiontransmission misalignments not only cause artifacts but also are common in stand-alone PET systems. Loghin et al. analyzed 1,177 diagnostic myocardial perfusion PET studies and found that $21 \%$ of them had artifactual defects due to an attenuation-emission mismatch (23). In stand-alone PET systems, this mismatch is due to patient movement between the transmission and emission scans. Integrated PET/CT scanners may lessen this problem by providing a faster transmission image from the CT component. However, the helical CT transmission acquisition is in another respect too fast, imaging only a fraction of a respiratory cycle. In short, whereas patient movement between the CT
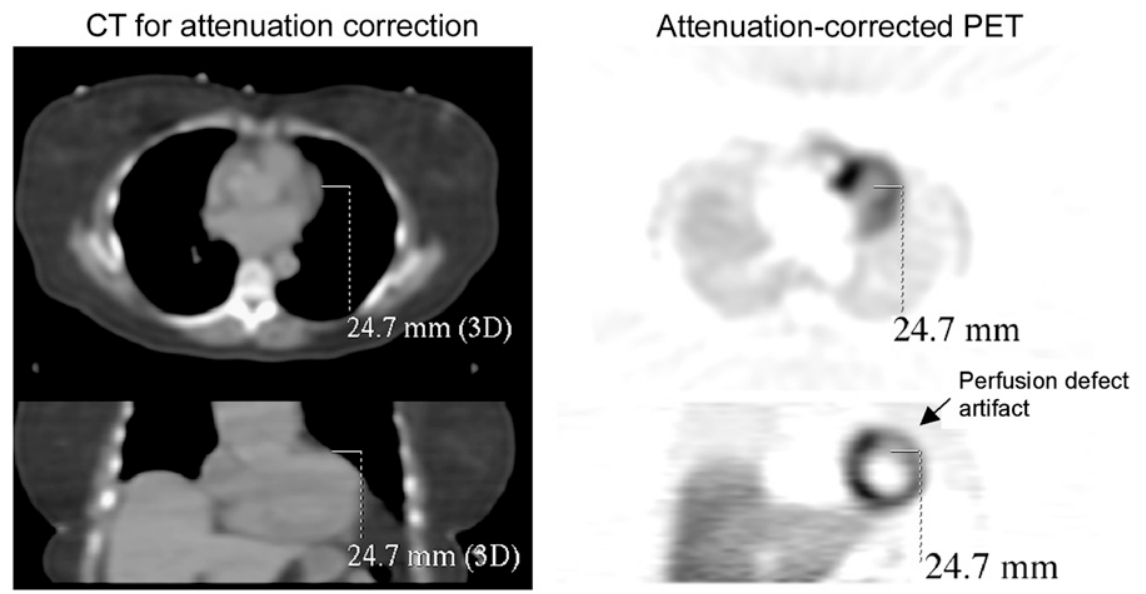

FIGURE 1. Cardiac PET/CT example of attenuation and emission scan mismatch, which often occurs along lateral free wall or right diaphragm. Axial and coronal images of CT scan and of attenuation-corrected $\mathrm{NH}_{3}$ PET scan show, as indicated with same solid line drawn on all slices, 24.7-mm mismatch at lateral wall. 
and PET scans remains a possible source of artifacts, the alignment is often further compromised by respiratory and cardiac motion.

\section{MATERIALS AND METHODS}

\section{Approaches to Attenuation Correction}

CT attenuation correction derived from the entire respiratory cycle will be more accurate than CT attenuation correction from only a single phase of the respiratory period. Several modern CT scanners support a "cine" acquisition mode that essentially collects multiple scans of the same region over time. We compared the use of conventional helical CT, the average of cine images, and the intensity maximum of cine images for attenuation correction. Figure 2 provides a simple example of the $3 \mathrm{CT}$ methods compared in this work. We collected cine images of a partially filled 2-cm-diameter sphere taped to a box that oscillated up and down during acquisition (period, $4 \mathrm{~s}$ ). The CT settings were a 0.8 -s rotation, a $2.5-\mathrm{mm}$ slice, $50 \mathrm{~mA}, 140 \mathrm{kVp}$, and $5 \mathrm{~s}$ per slice, effectively forming 5 consecutive images at each slice. The intensity-maximum image is formed from selecting the maximum value in each pixel across all time frames. This method is similar in concept to the maximum-intensity-projection method for the presentation of volumetric data. With maximum-intensity projection, the maximum pixel is selected across a projection through the object (across space domain). With intensity-maximum cine images, the maximum pixel is selected across the time domain. As a result, the intensity-maximum method will produce an image set that contains the minimum lung volume experienced through the normal course of respiration.

The PET acquisition can last 3-15 min, acquiring counts during an average of the respiratory motion. The use of the average cine CT image for attenuation correction offers one logical combination of the cine data. On the other hand, at any moment in time, the attenuation experienced by a single pair of coincident events is not the average of the body motion. Huesman et al. have shown that conventional PET transmission scanning, which collects an average of respiration and is similar in concept to the average of cine CT images, is inaccurate and less desirable than accurate phase matching of gated attenuation and emission data $(24,25)$. We do not have access to respiration-gated PET data in these cardiac studies to match the attenuation and emission phases. We
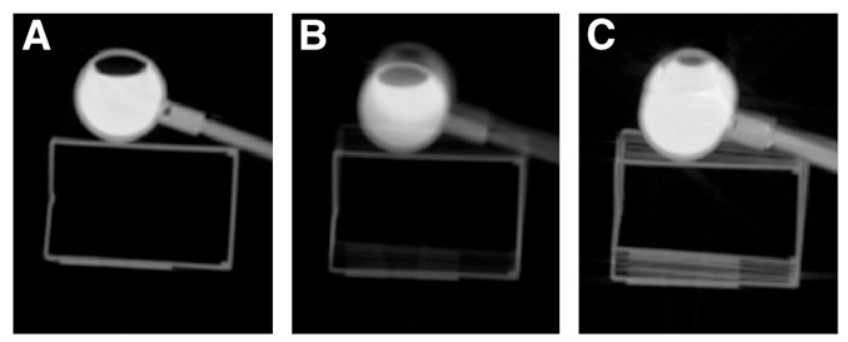

FIGURE 2. Transaxial slice through CT scans of oscillating box and water-filled sphere. (A) Image acquired in conventional helical mode (0.8-s rotation, $2.5-\mathrm{mm}$ slice, pitch of 1$)$. (B and $C)$ Images formed from cine CT images: average image of 5 time frames at same slice $(B)$ and intensity-maximum image of 5 time frames $(\mathrm{C})$. Because PET is performed during multiple complete respirations, attenuation correction derived from cine CT images may be more accurate than that derived from helical images. hypothesize that the intensity-maximum images will provide an alternative, reliable set of attenuation correction factors.

\section{Phantom Studies}

We tested the application of cine CT data for attenuation correction on measured phantom data from the GE DSTE scanner. We constructed a custom bed to simulate respiratory motion during acquisition. This bed consisted of a stationary portion supporting a moving platform and a drive mechanism powered by an electric motor that provided periodic axial translation $(3 \mathrm{~cm} / \mathrm{s})$. We placed a cardiac torso phantom (Data Spectrum Corp.) with no defect on the moving platform and filled it with ${ }^{18} \mathrm{~F}-\mathrm{FDG}$ with an activity concentration ratio of 5:1 for myocardial wall to background. We acquired conventional CT, cine CT, and PET data from this phantom with and without respiratory motion (periodic axial translation).

The cine CT acquisition collected 5 low-dose CT images (10 $\mathrm{mA}, 120 \mathrm{keV}, 0.8$-s rotation) at each slice over approximately $3 \mathrm{~s}$. We corrected the PET data with the conventional helical CT, an average of the 5 cine $\mathrm{CT}$ images, and an intensity maximum of the 5 cine CT images. These were compared with the ideal attenuation correction formed from PET and CT images acquired without motion for matched emission and attenuation correction factors. A weighted averaging of these ideal attenuation-corrected PET images was performed across multiple slices to induce respiratory motion to form a "true case."

\section{Patient Studies}

We have implemented cine CT for attenuation correction in the clinical workflow of our cardiac PET perfusion studies on the GE DSTE scanner. These ${ }^{13} \mathrm{~N}$ ammonia studies consist of a rest and pharmacologic stress portion for patients referred to the clinic with suspected coronary artery disease. We performed a cine CT acquisition (0.8-s rotation, 2.5-mm slice, $10 \mathrm{~mA}, 140 \mathrm{kVp}$ ) before and after both the rest and the stress PET acquisitions, for a total of 4 cine CT datasets with each patient. The protocol is outlined in Table 1. For both the rest and the stress study, we evaluated PET studies using attenuation correction from the average of the first cine, the intensity maximum of the first cine, the average of the second cine, and the intensity maximum of the second cine. We performed this protocol on 8 patients. We varied this protocol slightly for 2 additional patient studies (one containing no stress portion and the other containing no second cine for the stress portion), for a total of 74 attenuation-emission combinations.

The cine acquisition operates in step-and-shoot mode with the bed stationary for 1 cine duration, in which CT data are acquired for an entire respiratory cycle, before moving to the next bed location. In these patient studies, the cine duration was set on the basis of the respiratory period, which was determined from an optical real-time position monitor system (Varian Medical Systems). The cine duration for these patient studies ranged from 4 to $7 \mathrm{~s}$. The temporal sampling of the CT images can be adjusted by varying the time between $\mathrm{CT}$ images; for all studies, we fixed the time spacing to $0.8 \mathrm{~s}$ to match the rotation speed.

The attenuation-emission combinations were evaluated with a visual quality control process similar to the clinical task of evaluating the accuracy of the attenuation map. The attenuationcorrected image (from average or intensity maximum of cine CT images) and the attenuation-corrected PET image were viewed on a fusion workstation. The CT image window and level were set near 
TABLE 1

Protocol for Ammonia Cardiac Perfusion Study with Cine CT

\begin{tabular}{|c|c|c|}
\hline Steps & Time & Notes \\
\hline \multicolumn{3}{|l|}{ Prepare patient (14) } \\
\hline Acquire scout scan & $<1 \min$ & \\
\hline \multicolumn{3}{|l|}{ Rest study } \\
\hline \multicolumn{2}{|l|}{ Administer $\mathrm{NH}_{3}(370-740 \mathrm{MBq})$} & \\
\hline Acquire resting dynamic PET study & $4 \min$ & For myocardial blood flow quantitation \\
\hline Acquire resting cardiac gated PET study & $15 \min$ & For qualitative perfusion assessment \\
\hline Perform second resting cine CT attenuation correction & $<1 \min$ & \\
\hline Wait for isotope decay & $\sim 20 \mathrm{~min}$ & 40 min between injections \\
\hline \multicolumn{3}{|l|}{ Stress study } \\
\hline Administer dipyridamole & $4 \min$ & $0.56 \mathrm{mg} / \mathrm{kg}$ over $4 \mathrm{~min}$ \\
\hline Perform first stress cine CT attenuation correction & $<1 \min$ & \\
\hline Administer $\mathrm{NH}_{3}(555-925 \mathrm{MBq})$ & & \\
\hline Acquire stress dynamic PET study & $4 \min$ & For myocardial blood flow quantitation \\
\hline Acquire stress cardiac gated PET study & $15 \min$ & For qualitative perfusion assessment \\
\hline Perform second stress cine CT attenuation correction & $<1 \min$ & \\
\hline Total acquisition time & $\sim 80 \mathrm{~min}$ & \\
\hline
\end{tabular}

a lung window (window, 1,000; level, $-200 \mathrm{HU}$ ) to appropriately represent the tissue boundaries that are present in the attenuation map formed when the CT image is scaled to PET energy (19). Two cardiologists and one scientist independently evaluated the alignment of each attenuation-emission combination. The alignment was denoted "unacceptable" if there were sites of misalignment of more than $1 \mathrm{~cm}$ around the myocardial boundary leading to visible perfusion artifacts or if there were artifacts in the left ventricular region of the CT image leading to artifacts on the PET image. The attenuation-emission alignment was considered "acceptable" if the PET myocardial border and the CT myocardial border were aligned ( $<1-\mathrm{cm}$ deviation) along all surfaces.

\section{RESULTS}

\section{Phantom Studies}

Transaxial slices through the CT and PET images from the moving cardiac torso phantom appear in Figure 3. The first row presents the CT images that were used for attenuation correction of the PET images in the second row. A matching region of interest placed on the voxels of the myocardium in each PET image yielded the average \pm SD activity concentrations: true case, $71.9 \pm 7.6 \mathrm{kBq} / \mathrm{mL}$; helical attenuation correction, $23.8 \pm 14.1$; average cine attenuation correction, $32.5 \pm 9.5$; and cine intensitymaximum attenuation correction, $52.5 \pm 8.7$. The average and intensity-maximum cine $\mathrm{CT}$ images reduced the error in the defect by $20 \%$ and $60 \%$, respectively. The intensitymaximum method recovered the activity levels and reduced heterogeneity in the myocardium more than did the other methods.

\section{Patient Studies}

The use of cine CT acquisition for attenuation correction was added to our clinical workflow. A summary of the attenuation-emission alignment quality appears in Table 2. This evaluation compared 37 unique cine CT acquisitions
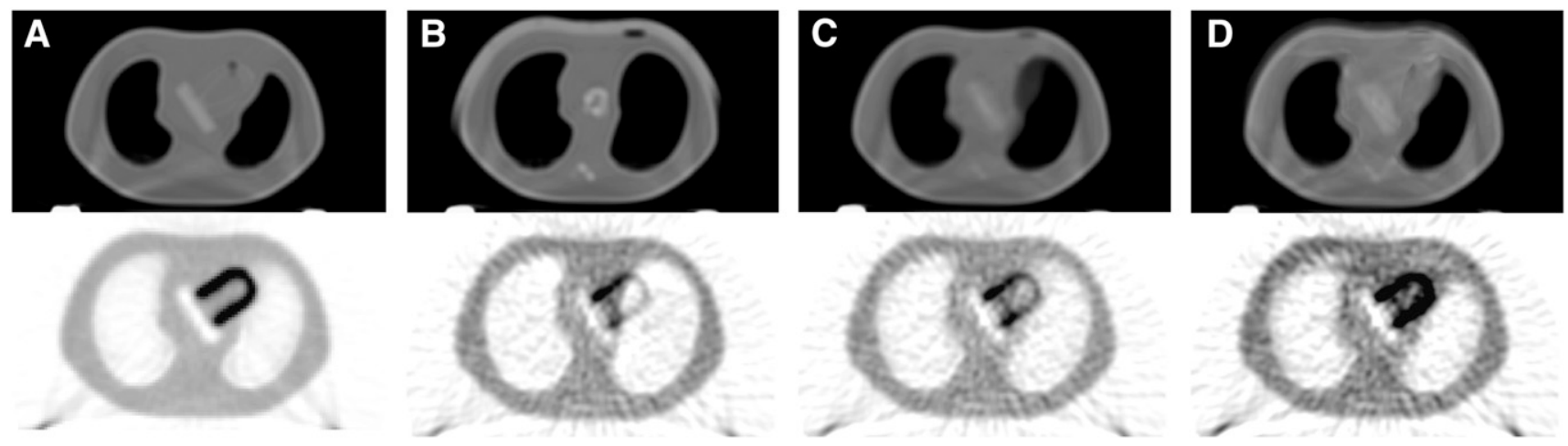

FIGURE 3. Comparison of attenuation correction techniques for cardiac PET, with CT images for attenuation correction at top and attenuation-corrected PET images at bottom. (A) PET/CT data acquired from stationary phantom processed to induce motion and generate the true case. (B-D) PET data acquired from moving phantom corrected with helical CT, as performed in current clinical practice $(B)$; with average cine CT (C); and with intensity-maximum cine CT (D). The proposed cine methods reduce bias and variance in myocardium. 
TABLE 2

Number of Unacceptable Attenuation-Emission Alignments for Rest, Stress, and Sum of Rest and Stress Cine CT

\begin{tabular}{|c|c|c|c|}
\hline $\begin{array}{c}\text { Cine CT } \\
\text { combination }\end{array}$ & $\begin{array}{c}\text { Rest } \\
(n=20)\end{array}$ & $\begin{array}{c}\text { Stress } \\
(n=17)\end{array}$ & $\begin{array}{c}\text { Total } \\
(n=37)\end{array}$ \\
\hline Average & $5 ; 3 ; 4$ & $5 ; 5 ; 4$ & $10 ; 8 ; 8$ \\
\hline Intensity maximum & $1 ; 2 ; 3$ & $2 ; 4 ; 1$ & $2 ; 6 ; 4$ \\
\hline
\end{tabular}

Data are for reviewer 1;reviewer 2;reviewer 3.

from 10 patients. The average of the cine CT image volumes provided acceptable alignment in $77 \%$ of the cases, and the intensity maximum of the same cine CT images provided acceptable alignment in $88 \%$ of the cases. No studies with unacceptable alignment from the intensitymaximum cine image had acceptable alignment with the cine average.

Figure 4 presents coronal images from the same PET study with attenuation correction from the average and from the intensity maximum of the cine images. The matching myocardial outline reveals the acceptable alignment with both methods. Figure 5 presents images from a study with an unacceptable average cine alignment and an acceptable intensity-maximum cine alignment. The outline drawn on the coronal images and the outline drawn on the transaxial images highlight the mismatched basal lateral myocardial boundary between the average cine image and the PET emission region.

Figure 6 presents average quantification values in different segments of the myocardium for a patient stress study with no perfusion defects. In this study, the average of the first cine acquisition had unacceptable alignment with the emission data, and all other methods had acceptable alignment. The intensity maximum of the cine data led to emission images with myocardial values approximately $2 \%-7 \%$ higher than those for emission images corrected with the average of the cine data. When images were normalized to have identical maximum values (similar to the qualitative task of visually assessing perfusion defects),

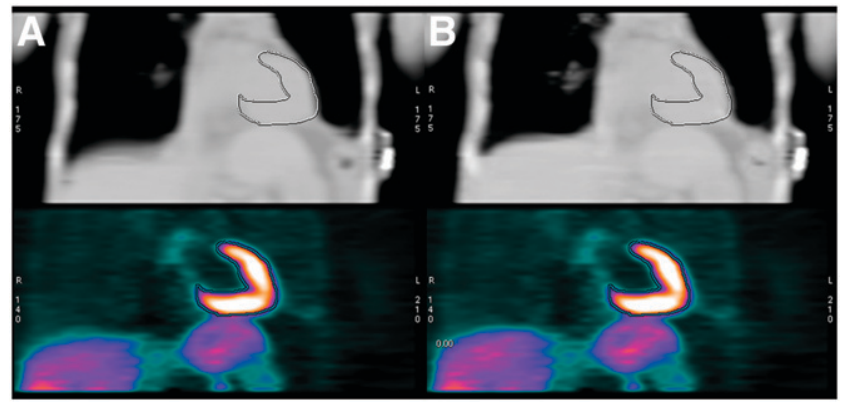

FIGURE 4. Coronal slice through patient example of aligned CT and PET images. At top are average (A) and intensity maximum (B) of 1 set of cine CT data. Top row was used for attenuation correction of PET images in bottom row.

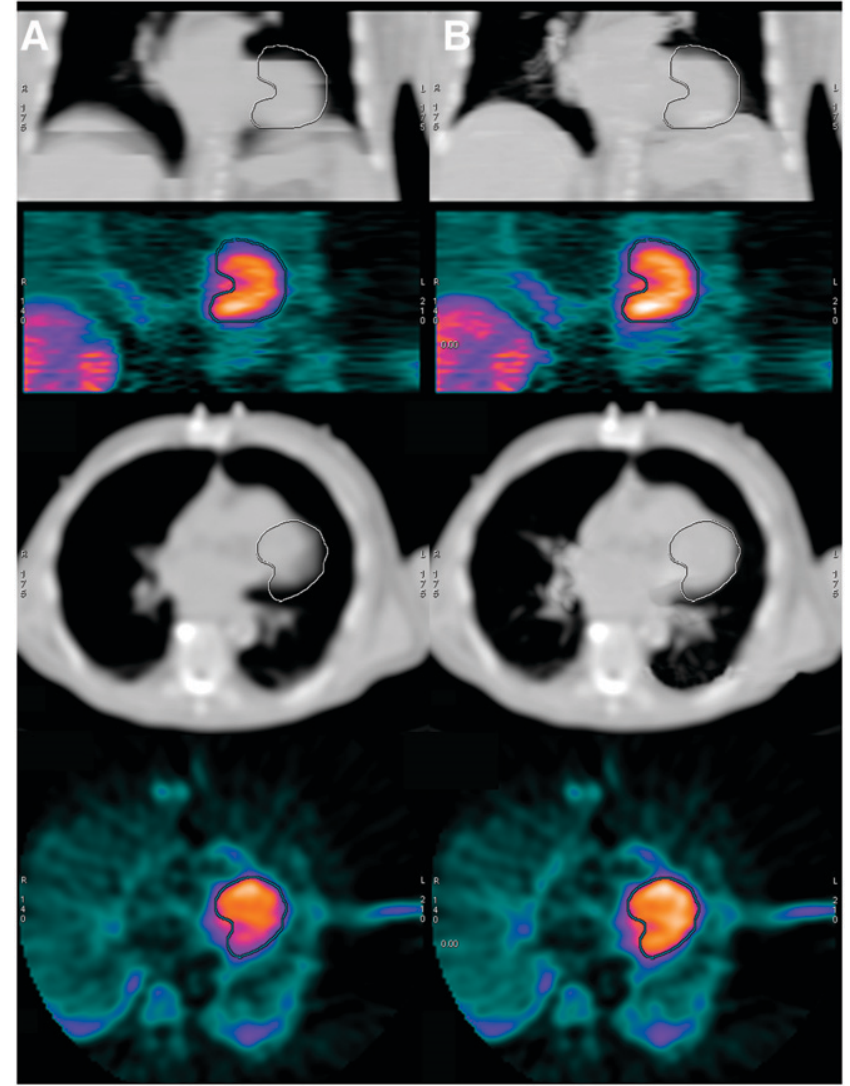

FIGURE 5. Patient example of unacceptable alignment from average of cine CT images (column A) and acceptable alignment from intensity maximum of cine CT images (column B). Top row contains coronal slice of CT used for attenuation correction of PET image seen in second row from top. Third row from top contains transaxial slice of same CT image used for attenuation correction of PET image seen in bottom row. Identical myocardial outline is drawn on all coronal views, and separate identical outline is drawn on all transaxial views.

the intensity-maximum and average attenuation correction methods led to similar global levels when the alignment was acceptable. In this example, when the alignment was unacceptable (average of first cine), the anterior and anterolateral segments of the heart showed a clear deficit, which was partially resolved with the intensity maximum of the same cine data and completely resolved with the aligned second cine acquisition.

\section{DISCUSSION}

The phantom study supports the use of cine CT for attenuation correction, showing reduced bias and reduced potential for artifactual perfusion defects due to erroneous heterogeneity. Furthermore, in this study, the intensity maximum of the cine data for attenuation correction appeared to outperform the average of the cine data. The CT images (Fig. 4) revealed that averaging the cine data can cause motion-blurred boundaries that, when translated to PET energies, will have erroneous values leading to undercorrected attenuation in these localized (clinically 


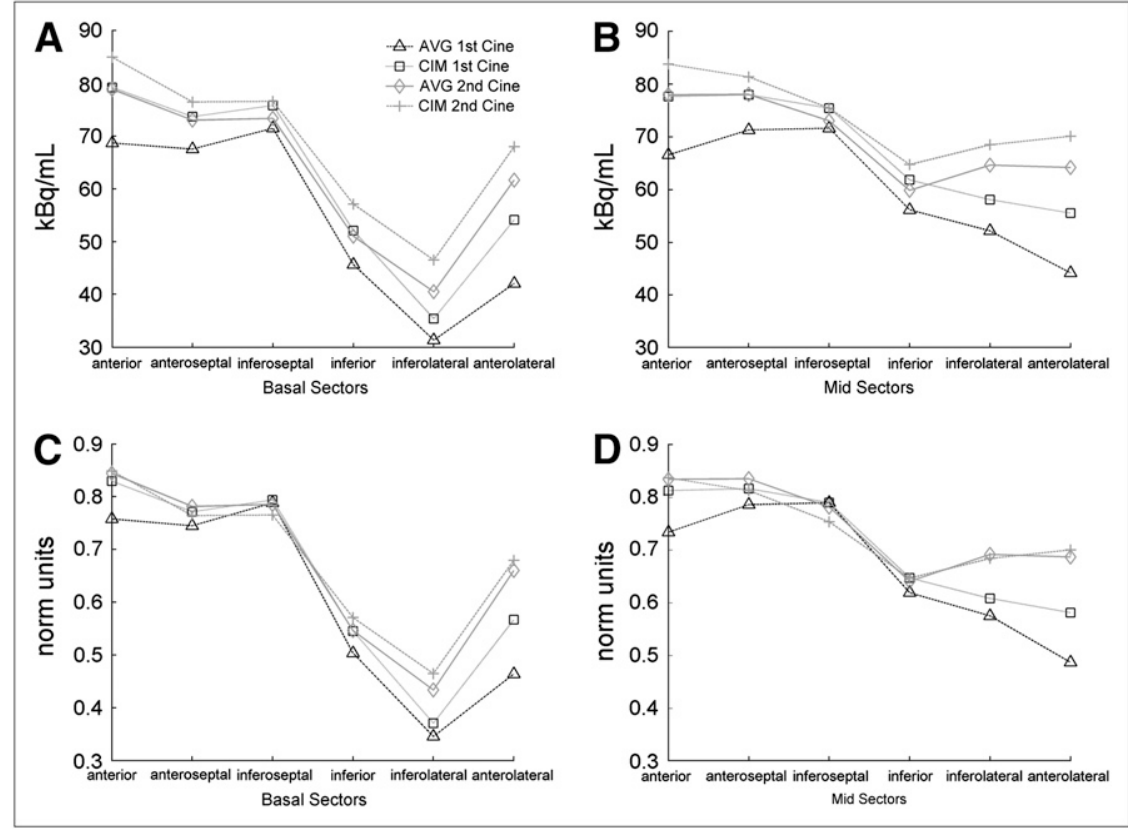

FIGURE 6. Average quantification in myocardium along circumferential profiles through short-axis view of basal segments ( $A$ and $C$ ) and mid segments ( $B$ and $D$ ) for identical emission data attenuation-corrected with different methods: plots of average $\mathrm{kBq} / \mathrm{mL}$ in segments ( $A$ and $B$ ) and plots of same segments with units normalized (norm) such that images from all methods have same maximum value $(C$ and $D)$. This patient scan was classified as normal, with unacceptable alignment for average of first cine and with acceptable alignment for all other methods. meaningful) regions. The intensity maximum of the cine CT data did not have blurred boundaries. Along the boundary of the mediastinum and lung, the intensity-maximum image provided the maximal motion extent of the muscle/ soft tissue. Therefore, whereas the intensity-maximum image overestimated the boundary location, it did provide constant (not blurred) coefficients for correcting muscle/ soft-tissue attenuation along the myocardium. In short, the average of the cine CT can lead to undercorrected attenuation in the PET myocardium (possible defect artifacts) and the intensity maximum of the cine CT can cause overcorrected attenuation in regions outside the PET myocardium (possible high-activity artifacts). Considering the fact that errors from attenuation correction are highly localized to the regions with errors, intensity-maximum attenuation correction may be preferable to average attenuation correction for cardiac imaging.

In the phantom experiment, conventional helical CT imaged the phantom at approximately mid expiration but could have captured the phantom at any location in the cycle (similar to no breath coaching). Given the geometry of the phantom, helical CT would have yielded better results if the scan had captured the phantom at full expiration (cranial extreme translation). Breath coaching could be used to improve the potential accuracy of the helical acquisition. But, as this example highlights, when helical CT is performed at a nonoptimal respiratory phase, the attenuation-emission misalignment can cause artifactual perfusion defects. And, as stated earlier, coaching patients to hold their breath in the optimal respiratory phase is a challenge and relies on patient compliance.

Figure 5 shows a mismatch in a patient study from the average cine CT along the basal lateral portion of the myocardium. This is a common region for a mismatch, considering that respiration causes the mediastinum to move primarily along the craniocaudal axis. Consequently, the lateral portion of the heart on the PET image can appear in the lung space of the CT image, leading to undercorrected attenuation in this region and reduced confidence in the values along the lateral myocardial wall on the PET image. The use of the intensity-maximum image for attenuation correction (with $12 \%$ of the cases having unacceptable alignment) had fewer cases of unacceptable alignment than did the use of average cine for attenuation correction; these cases were still misaligned because of patient movement (shifting body position) and aberrant breathing patterns between the CT acquisition and the PET acquisition. Although a more thorough analysis of quantification is warranted, an initial analysis showed that when images were normalized, aligned studies had similar quantitation whether attenuation correction was performed with the intensity-maximum cine image or with the average cine image.

The coronal CT slice in Figure 5 revealed a common artifact on the average cine images. These images were prone to mismatches at the edges of the CT axial fields of view because of a motion mismatch between each bed location in the step-and-shoot acquisition. The intensitymaximum images were less prone to the motion mismatch because only the extreme tissue boundaries (instead of motion-blurred boundaries) were retained. One method for reducing these mismatches in the average image was to decrease the temporal spacing between the cine CT images by generating more CT images for each bed location. We used 0.8-s spacing between images, and a 0.8-s rotation speed, for essentially no data overlap on each CT image. 
FIGURE 7. Coronal slices through average of cine CT data (A), intensitymaximum image $(B)$, and weighted combination of average and intensitymaximum image (C). Weighted-

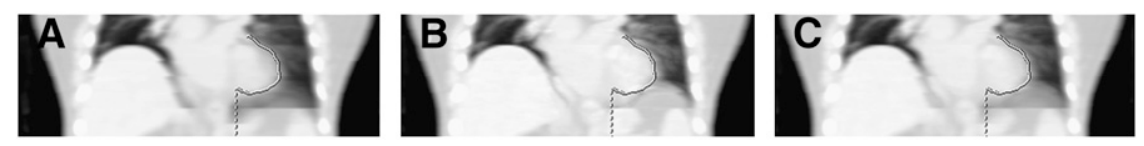
combination image reduces some of the stair-step axial-field-of-view artifacts present on average image and shows a potentially more accurate boundary extent than does intensity-maximum image.

We could reduce the spacing to form more CT images at each location to provide a potentially smoother average image. Furthermore, a faster rotation speed would avoid artifacts within a single CT image due to structures moving into and out of the slice location during the acquisition of a single image.

This work evaluated attenuation correction with either the average or the intensity-maximum image. Another possible method for attenuation correction is to use a weighted combination of the average and intensity-maximum images as $(1-\mathrm{WF})$ average $+(\mathrm{WF})$ intensity maximum, where WF is the weighting factor ( 0 to 1$)$. This combination could offer a compromise between the inaccurate blurred boundaries on the average image and the inaccurate oversized boundaries on the intensity-maximum image. Figure 7 presents a possible combination of average and intensity-maximum images (equal weighted average of 2 images, with a WF of 0.5 ).

Multiple CT scans at 1 slice location raise concern about radiation dose. In cardiac PET, only 1 PET axial field of view ( 1 bed location) is necessary for each image acquisition. A $15-\mathrm{cm}$ coverage for the $10-\mathrm{mA}, 140-\mathrm{kVp}, 5.6-\mathrm{s}$ cine $\mathrm{CT}$ acquisition resulted in an effective dose of around $2 \mathrm{mSv}$. Even though individual 10-mA cine images are dramatically noisier than conventional diagnostic studies, attenuation maps for PET are highly smoothed. Likewise, the average and intensity-maximum combination of noisy images have improved signal-to-noise properties because they are derived from multiple images at each location. We chose $10 \mathrm{~mA}$ because it is the present lower limit on the CT tube; this level could be reduced if the CT scanner supported lower currents. The effective dose could also be reduced if the CT acquisition could be performed on only the portion of the image volume with myocardium, which is often smaller than the 15-cm PET field of view. The cine CT effective dose is analogous to that of a normal helical CT scan with the same coverage using a tube current of $60 \mathrm{mAs}$, which is a lower current (and dose) than is used in many centers. In ${ }^{82} \mathrm{Rb}$ imaging, where the effective dose for a rest-stress study is in the range of $10-15 \mathrm{mSv}$ (26), the addition of 4 cine $\mathrm{CT}$ acquisitions adds approximately $50 \%$ more radiation. For ${ }^{13} \mathrm{~N}$ imaging, in which the PET acquisitions have an effective dose of around $3 \mathrm{mSv}$, the total study dose including $4 \mathrm{CT}$ acquisitions is similar to that of a conventional ${ }^{99 \mathrm{~m}} \mathrm{Tc}$ imaging study.

Alternative CT acquisitions for imaging respiratory motion have been proposed and evaluated. Slow-scan helical $\mathrm{CT}$ acquisition uses a slow gantry rotation to capture a respi- ratory cycle in 1 rotation. This acquisition collects inconsistent data from different phases of respiration and is therefore prone to artifacts in motion regions of the image (27). Another approach uses a helical acquisition with a low pitch (slow table translation). Pan has shown that low-pitch CT offers slightly faster acquisition times than cine CT but less desirable dose efficiency and slice thickness properties (28).

\section{CONCLUSION}

Cine CT offers an alternative to helical CT to compensate for respiratory motion in attenuation correction of cardiac PET studies. Phantom studies suggested that the average and the intensity maximum of the cine CT images can reduce potential respiration-induced misalignment errors in attenuation correction. Patient studies revealed that cine CT images provide acceptable attenuation-emission alignment in most cases. The studies also showed that the intensitymaximum image is aligned more often than is the average cine image, suggesting that the former offers more robust attenuation correction than the latter.

\section{ACKNOWLEDGMENTS}

We thank Diane Miesbauer, Joni Hanson, Nancy Jeffers, and Dennis Hurley of GE Healthcare for conversations on data acquisition. We also thank Kevin Berger of Michigan State University for conversations on cardiac imaging. This work was supported by NIH grants CA-74135 and CA115870-01 and by GE Healthcare.

\section{REFERENCES}

1. Becker CR, Knez A, Ohnesorge B, Schoepf UJ, Reiser MF. Imaging of noncalcified coronary plaques using helical CT with retrospective ECG gating. AJR. 2000;175:423-424.

2. Schroeder S, Kopp AF, Baumbach A, et al. Noninvasive detection of coronary lesions by multislice computed tomography: results of the new age pilot trial. Catheter Cardiovasc Interv. 2001;53:352-358.

3. Fitzpatrick GM, Wells G. Mis-registration between emission and transmission data in cardiac PET/CT. In: IEEE Nuclear Science Symposium Conference Record. 2003;5:3048-3052.

4. Chin BB, Nakamoto Y, Kraitchman DL, Marshall L, Wahl R. PET-CT evaluation of 2-deoxy-2-[ $\left.{ }^{18} \mathrm{~F}\right]$ fluoro-D-glucose myocardial uptake: effect of respiratory motion. Mol Imaging Biol. 2003;5:57-64.

5. Goerres G, Burger C, Kamel E, et al. Respiration-induced attenuation artifact at PET/CT: technical considerations. Radiology. 2003;226:906-910.

6. Beyer T, Antoch G, Blodgett T, Freudenberg LF, Akhurst T, Mueller S. Dualmodality PET/CT imaging: the effect of ACF-artifact, respiratory motion on combined image quality in clinical oncology. Eur J Nucl Med Mol Imaging. 2003;30:588-596. 
7. Goerres GW, Kamel E, Heidelberg TN, Schwitter MR, Burger C, Schulthess GK. PET-CT image co-registration in the thorax: influence of respiration. Eur J Nucl Med Mol Imaging. 2002;29:351-360.

8. Nakamoto Y, Osman M, Cohade C, et al. PET/CT: comparison of quantitative tracer uptake between germanium and CT transmission attenuation-corrected images. J Nucl Med. 2002;43:1137-1143.

9. Pan T, Mawlawi O, Nehmeh SA, et al. Attenuation correction of PET images with respiration-averaged CT images in PET/CT. J Nucl Med. 2005;46:1481-1487.

10. Cook R, Carnes G, Lee T, Wells G. 4D CT for respiratory gated attenuation corrections in canine cardiac PET imaging. In: IEEE Nuclear Science Symposium Conference Record. 2005;4:2408-2412.

11. Shoder J, Schelbert H. PET for the assessment of myocardial viability. In: Dilsizian V, ed. Myocardial Viability: A Clinical and Scientific Treatise. Armonk, NY: Futura; 2000:391-418.

12. Schelbert HR. ${ }^{18} \mathrm{~F}$-Deoxyglucose and the assessment of myocardial viability. Semin Nucl Med. 2002;32:60-69.

13. Knuuti J. Clinical cardiac PET in the future. Eur J Nucl Med Mol Imaging. 2004;31:467-468.

14. Hutchins G, Schwaiger M, Rosenspire K, Krivokapich J, Schelbert H, Kuhl D. Noninvasive quantification of regional blood flow in the human heart using N-13 ammonia and dynamic positron emission tomographic imaging. J Am Coll Cardiol. 1990;15:1032-1042.

15. Beanlands RS, Muzik O, Melon P, et al. Noninvasive quantification of regional myocardial flow reserve in patients with coronary atherosclerosis using nitrogen13 ammonia positron emission tomography: determination of extent of altered vascular reactivity. J Am Coll Cardiol. 1995;26:1465-1475.

16. Schwaiger M, Ziegler S, Bengel F. Assessment of myocardial blood flow with PET. In: Shah P, ed. Imaging in Cardiovascular Disease. Philadelphia, PA: Lippincott Williams and Wilkins; 2000:195-212.

17. Muzik O, Duvernoy C, Beanlands FC, et al. Assessment of diagnostic performance of quantitative flow measurements in normal subjects and patients with angiographically documented coronary artery disease by means of nitrogen-13 ammonia and positron emission tomography. J Am Coll Cardiol. 1998;31:534-540.
18. Boyd HL, Gunn RN, Marinho NV, et al. Non-invasive measurement of left ventricular volumes and function by gated positron emission tomography. Eur J Nucl Med. 1996;23:1594-1602.

19. Kinahan PE, Hasegawa BH, Beyer T. X-ray-based attenuation correction for positron emission tomography/computed tomography scanners. Semin Nucl Med. 2003;33:166-179.

20. McCord M, Bacharach S, Bonow R, Dilsizian V, Cuocolo A, Freedman N. Misalignment between PET transmission and emission scans: its effect on myocardial imaging. J Nucl Med. 1992;33:1209-1214.

21. Ter-Pogossian M, Bergmann S, Sobel B. Influence of cardiac and respiratory motion on tomographic reconstructions of the heart: implications for quantitative nuclear cardiology. J Comput Assist Tomogr. 1982;6:1148-1155.

22. Bacharach SL, Bax JJ, Case J, et al. American Society of Nuclear Cardiology practice guidelines: PET myocardial glucose metabolism and perfusion imaging. J Nucl Cardiol. 2003;10:543-556.

23. Loghin C, Sdringola S, Gould K. Common artifacts in PET myocardial perfusion images due to attenuation-emission misregistration: clinical significance, causes, and solutions. J Nucl Med. 2004;45:1029-1039.

24. Reutter B, Klein G, Huesman R. Respiration-compensated cardiac PET attenuation correction via automated 4-D segmentation of gated transmission images [abstract]. J Nucl Med. 1997;38(5 suppl):203P.

25. Huesman RH, Klein G, Reutter BW, Budinger TF. Preliminary Studies of Cardiac Motion in Positron Emission Tomography: Berkeley, CA: Lawrence Berkeley National Laboratory; 2001.

26. Einstein AJ, Moser KW, Thompson RC, Cerqueira MD, Henzlova MJ. Effect of proposed new ICRP guidelines on effective dose for SPECT and PET myocardial perfusion studies. J Nucl Cardiol. 2006;13(suppl):S15-S16.

27. Mori S, Kanematsu N, Mizuno H, Sunaoka M, Endo M. Physical evaluation of CT scan methods for radiation therapy planning: comparison of fast, slow and gating scan using the 256-detector row CT scanner. Phys Med Biol. 2006;51: $587-600$.

28. Pan T. Comparison of helical and cine acquisitions for $4 \mathrm{D}-\mathrm{CT}$ imaging with multislice CT. Med Phys. 2005;32:627-634. 